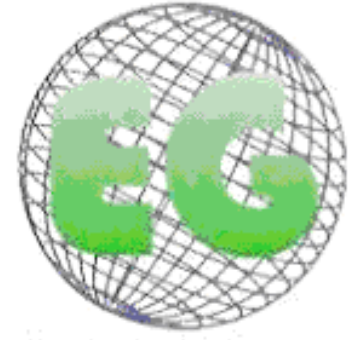

ISSN 1695-6141 N028

\title{
Sistematización de la asistencia de enfermería: enfoque en un paciente portador de úlcera venosa. Estudio de caso.
}

Systematization of nurse care: focus on a patient with venous ulcer. Case study.

\author{
${ }^{*}$ Marques Frota, N. ${ }^{* *}$ Ramalho Rolim, L., ${ }^{* * *}$ Mesquita Melo, E., ${ }^{* * *}$ de \\ Almeida, NG. ${ }^{* * * * B}$ Barbosa, IV, ${ }^{* * *}$ de Almeida, DT.
}

\begin{abstract}
*Enfermera. Alumna del Curso de Especialización en Enfermería del Trabajo por la Universidad Estadual de Ceará (UECE). E.mail: natashafrota @hotmail.com ${ }^{* *}$ Enfermera. Alumna del Curso de Especialización en Enfermería en Nefrología por la Universidad Estadual de Ceará (UECE). ****Doctora en Enfermería por la Universidad Federal de Ceará (UFC). Profesora de la UNIFOR. ${ }^{* * * * E s t u d i a n t e ~ d e ~}$ Enfermería de la UFC. *****Máster en enfermería por la UFC. Profesora de la UNFOR. Brasil.
\end{abstract}

Palabras clave: Enfermería; Úlcera de pierna; Cuidados de enfermería.

Keywords: Nursing; Leg ulcer; Nursing care.

\section{RESUMEN}

Las úlceras venosas representan cerca de $70 \%$ a $90 \%$ de los casos de úlceras de pierna, estando asociadas a la insuficiencia venosa crónica. El estudio tuvo como objetivo demostrar la importancia de la asistencia de enfermería al paciente portador de úlcera venosa. Estudio de caso, desarrollado en una institución hospitalaria, en Fortaleza-Ceará-Brasil, durante el mes de marzo de 2010, con un paciente portador de insuficiencia venosa, presentando úlcera venosa en miembro inferior izquierdo. Para la obtención de los datos se utilizó como base la Sistematización de la Asistencia de Enfermería (SAE). Fue realizada la entrevista con el paciente y acompañante, consulta al prontuario y examen físico del paciente, siendo este aclarado en cuanto a los objetivos e importancia del estudio. Fueron levantados los problemas e identificados los siguientes diagnósticos de enfermería según la Taxonomía de la NANDA 2007-2008: integridad tisular perjudicada relacionada a la circulación afectada y factores mecánicos; deambulación afectada relacionada a perjuicio cognitivo, neuromuscular y musculoesquelético y fuerza muscular insuficiente; dolor crónico relacionado con incapacidad física crónica; ansiedad relacionada con estado de salud afectado; y disturbio en la imagen corporal relacionado con lesión. Posteriormente fueron planificadas las intervenciones de enfermería. Es fundamental el papel de la enfermería en los cuidados al paciente portador de úlcera venosa, por tratarse de una enfermedad crónica, con recuperación lenta, con posibilidad de recidivas e implicaciones importantes en la vida del paciente. 


\begin{abstract}
Venous ulcers account for about $70 \%$ to $90 \%$ of leg ulcer cases and are associated with chronic venous insufficiency. The study was aimed to demonstrate the importance of nursing care towards patients with venous ulcers. The case study was developed in a hospital in Fortaleza, Ceara, Brazil, during the month of March 2010, with a patient with venous insufficiency and venous ulcers in the left leg. To obtain the data the Systematization of Nursing Care (SNC) was used as a basis. An Interview was performed with the patient and attendant, with consultations to medical records and the physical examination of the patient, who was informed about the objectives and importance of the study. The problems were raised and identified following nursing diagnoses according to NANDA Taxonomy 2007-2008: impaired tissular integrity related to impaired circulation and mechanical factors, impaired ambulation related to cognitive impairment, neuromuscular and musculoskeletal and muscular strength insufficient; chronic pain related to chronic physical disability, anxiety related to impaired health; and body image disturbance in connection with the injury. Later were planned nursing interventions. It is essential to the role of nurse care to patients with venous ulcers because it is a chronic disease with a slow recovery and with the possibility of recurrence and potential major implications for the patient's life.
\end{abstract}

\title{
INTRODUCCIÓN
}

El paciente portador de heridas necesita un abordaje multiprofesional con énfasis en los diversos aspectos que interfieren en el desarrollo y evolución de la lesión. Así, resulta esencial el trabajo de un equipo compuesto por médico, enfermero, nutricionista, fisioterapeuta y farmacéutico, para que sean considerados todos los factores relacionados con la cicatrización, tanto asociados al paciente como a la propia lesión.

Herida es toda y cualquier ruptura de la integridad de un tejido u órgano, pudiendo alcanzar desde la epidermis, que es la capa más externa de la piel, hasta estructuras más profundas como fascias, músculos, aponeurosis y órganos cavitarios ${ }^{(1)}$.

Las curas constituyen una forma de tratamiento de las heridas cutáneas y su elección depende de factores intrínsecos y extrínsecos. El tratamiento de las heridas cutáneas es dinámico y depende, a cada momento, de la evolución de las fases de cicatrización (2)

En este contexto, el enfermero, como profesional responsable de la realización de la cura, debe conocer de forma profunda los aspectos referentes a las heridas, tales como las fases de cicatrización, tejidos presentes en el lecho de las mismas y factores relacionados con su evolución, para que pueda elegir el producto más adecuado para usar en determinada lesión.

Los profesionales de salud se encuentran comúnmente con úlceras de miembros inferiores causadas por insuficiencia venosa y arterial. La úlcera arterial presenta lesión con bordes lisos, profunda y de coloración pálida, generalmente sin tejido de granulación, pudiendo ocurrir exudado mínimo, gangrena o necrosis. La úlcera venosa es una lesión de forma irregular, pero con bordes definidos y poco profunda. El centro de la herida expuesta tiene tejido de granulación, si no está infectada, exudado moderado o abundante ${ }^{(3)}$.

Las úlceras venosas representan cerca de $70 \%$ a $90 \%$ de los casos de úlceras de pierna, estando asociadas a la insuficiencia venosa crónica, por causa de una incompetencia valvular, asociada o no a la obstrucción del flujo venoso ${ }^{(4)}$. Las mismas son desencadenadas por diversos factores incluyendo alteraciones vasculares, 
metabólicas y hematológicas, hipertensión venosa, varices secundarias, edema, alteraciones tróficas, neuropatía, linfedema, traumas, osteomielitis crónica, artritis reumatoide, anemia falciforme, vasculitis, tumores cutáneos y enfermedades infecciosas crónicas ${ }^{(4 ; 5)}$.

El diagnóstico y el control adecuados de las úlceras venosas comienzan con una comprensión del sistema venoso de los miembros inferiores. Las venas y los músculos de la región inferior de la pierna constituyen una unidad fisiológica denominada musculatura-pantorrilla. La activación de la bomba musculaturapantorrilla durante actividades, fuerza la sangre de las venas profundas en dirección al corazón, resultando en caída de la presión inferior del sistema venoso profundo, facilitando el movimiento de la sangre del sistema superficial hacia el profundo por medio de una serie de venas comunicantes ${ }^{(3)}$.

La lesión de cualquier componente de la bomba musculatura-pantorrilla puede generar un reflujo, denominado insuficiencia venosa. Una bomba musculaturapantorrilla con funcionamiento deficiente no reduce satisfactoriamente la presión en el sistema venoso profundo, generando hipertensión venosa ${ }^{(3)}$.

Generalmente las úlceras venosas se manifiestan en el tercio inferior de la pierna, un poco por encima del maléolo interno, a veces en el externo, en el dorso del pie y/o más raramente en el tercio medio de la pierna ${ }^{(7)}$. Estas úlceras son comunes en la población adulta, sin embargo, esta inadecuación del funcionamiento del sistema venoso está muy presente en los ancianos, siendo la frecuencia superior al $4 \%$ entre ancianos de más de 65 años ${ }^{(5)}$.

Las úlceras venosas pueden ser clasificadas como heridas crónicas, exigiendo, generalmente, un periodo largo de ingreso hospitalario y tratamiento ambulatorio. Además, es común que el paciente presente recidivas después de la resolución de la úlcera, lo que puede estar asociado a la falta de cuidados más dirigidos a la prevención.

El principal motivo de las recidivas de las úlceras venosas es la negligencia del paciente en relación a las medidas preventivas, como el uso de medias de compresión, para facilitar el retorno venoso al corazón. Estas medias deben ser aplicadas por la mañana antes del paciente deambular y sacadas antes de acostarse. Los portadores de enfermedad venosa deben usar las medias toda la vida para el mantenimiento del tratamiento de la insuficiencia venosa ${ }^{(4)}$.

El enfermero representa un punto de apoyo para el paciente portador de estas úlceras, correspondiendo al mismo intervenir, cuidar e incentivar al individuo a utilizar sus mejores posibilidades de respuesta a los factores estresantes. Vale destacar la importancia de las evaluaciones constantes de la integridad cutánea del paciente y de la intervención eficaz de enfermería ${ }^{(8)}$.

Considerando la problemática de las úlceras venosas, que pueden traer consecuencias diversas a la vida de su portador, el estudio se hace relevante, por poner en foco la asistencia de enfermería al paciente con úlcera venosa, posibilitando una intervención dirigida a las necesidades afectadas del paciente y contribuyendo a su recuperación. Así, el estudio tuvo como objetivo demostrar la importancia de la asistencia de enfermería al paciente portador de úlcera venosa. 


\section{METODOLOGÍA}

Se realizó un estudio descriptivo, del tipo estudio de caso, con abordaje cualitativo, que se fundamenta en el análisis descriptivo de un determinado evento, en el cual el investigador no se implica en la evaluación de los datos obtenidos ${ }^{(9)}$.

Se buscó describir el caso clínico de un paciente portador de úlcera venosa, enfatizando, esencialmente. la asistencia de enfermería, levantando diagnósticos y estableciendo las intervenciones de enfermería.

Para la realización del estudio, utilizamos como campo una institución hospitalaria de la red municipal de salud, localizada en Fortaleza-Ceará-Brasil, referencia en atención de urgencia y emergencia, con enfoque principal en emergencias quirúrgicas y traumatológicas.

El estudio fue desarrollado durante el mes de marzo de 2010, como parte de las actividades de la disciplina Prácticas Supervisadas II, del Curso de Enfermería, de la Universidad de Fortaleza.

El sujeto del estudio fue un paciente internado en la referida institución, con diagnóstico de insuficiencia venosa, presentando úlcera venosa en miembro inferior izquierdo (MII).

La opción por este paciente se basó en el interés por la enfermedad, dada su cronicidad, la cual necesita el seguimiento del enfermero, englobando cuidados con la herida y orientación para el autocuidado y prevención de recidivas.

Para la obtención de los datos del estudio, se utilizó como base la Sistematización de la Asistencia de Enfermería (SAE), que debe ser construida a partir de un referencial teórico que oriente su aplicación, consistiendo en cinco fases secuenciales e interrelacionadas: levantamiento de los datos (anamnesis y examen físico); diagnóstico de enfermería; planificación; implementación, y evaluación ${ }^{(10)}$.

Fue realizada la entrevista con el paciente, complementada con los datos referidos por el acompañante y la consulta al prontuario. El examen físico fue realizado de forma minuciosa, buscando identificar características definidoras que llevasen al establecimiento de los diagnósticos de enfermería.

Los datos fueron analizados siguiendo los pasos de la SAE propuesta por AlfaroLefevre (10). Así, fueron levantados los problemas, con la identificación de los diagnósticos de enfermería según la Taxonomía de la NANDA 2007-2008 ${ }^{(11)}$ y posteriormente fueron planificadas las intervenciones de enfermería, las cuales fueron implementadas y evaluadas de forma continua.

El trabajo contempló los principios contenidos en la Resolución 196/96, del Consejo Nacional de Salud, que preconiza normas y principios para el desarrollo de investigaciones con seres humanos ${ }^{(12)}$.

Para ello, el paciente fue informado en cuanto a los objetivos e importancia del estudio, siendo su participación voluntaria, con libertad de desistir en cualquier momento, sin perjuicio en su atendimiento en la institución. Se le garantizó el 
anonimato en cuanto a su identidad y la utilización de los resultados únicamente para fines científicos.

\section{RESULTADOS}

\section{Relato de caso}

J.V.S., 83 años, sexo masculino, casado, analfabeto, jubilado. Buscó atendimiento presentando úlcera venosa en MII. Relata que, hace aproximadamente 40 años sufrió una quemadura en el miembro inferior izquierdo, siendo frecuente la inflamación, hasta convertirse en una herida abierta como se encuentra hoy. Fumó durante muchos años, aunque lo dejó hace más o menos cinco años. Niega diabetes, etilismo e hipertensión. Demostró ansiedad antes de la cirugía, realizada en 29/03/2010. Otras cirugías fueron realizadas para la preparación del área para el injerto cutáneo. Durante el ingreso se realizaron cuidados para la mejora de la lesión: cura en días alternos, siendo utilizado suero fisiológico (SF) a 0,9\%, alginato de calcio, ácidos grasos esenciales (AGE) y tela no adherente. Se queja de dolor en la cura y cuando la pierna queda baja. Examen físico: Consciente, orientado, comunicativo (Escala de Coma de Glasgow=15). Encamado, no deambula, utilizando como ayuda silla de ruedas. Hemodinámicamente estable. Respiración espontánea, sin ayuda de $\mathrm{O}_{2}$. Ausculta pulmonar y cardiaca sin alteraciones. Miembros superiores (MMSS) sin edemas, normohidratados, acceso venoso periférico en miembro superior izquierdo (MSI), perfusión periférica mantenida. Abdomen: plano, flácido, ruidos hidroaéreos presentes. Acepta parcialmente la dieta ofrecida. Miembros inferiores (MMII) con perfusión periférica mantenida, curativo oclusivo en MII. Eliminaciones fisiológicas presentes. Exámenes realizados: hemograma completo; urea; creatinina; glicemía; coagulograma; electrocardiograma; y rayo-x de tórax. La terapia farmacológica incluyó: antibiótico, analgésico, antitérmico, procinético, anti ulceroso, anticoagulante y antiagregante plaquetário, además de la orientación al paciente de mantener los MMll elevados a $40^{\circ}$.

Sistematización de la asistencia de enfermería

\begin{tabular}{|c|c|c|}
\hline $\begin{array}{c}\text { Problemas de } \\
\text { enfermería }\end{array}$ & $\begin{array}{l}\text { Diagnósticos de } \\
\text { enfermería }\end{array}$ & Intervenciones \\
\hline $\begin{array}{l}\text { Herida en miembro inferior } \\
\text { izquierdo }\end{array}$ & $\begin{array}{l}\text { Integridad tisular } \\
\text { perjudicada relacionada a la } \\
\text { circulación afectada y } \\
\text { factores mecánicos. }\end{array}$ & $\begin{array}{l}\text { Administrar medicamentos } \\
\text { conforme a prescripción médica. } \\
o \text { Monitorear posicionamiento } \\
\text { adecuado del paciente. } \\
\text { o Renovar curativo, eligiendo el } \\
\text { producto adecuado de } \\
\text { estableciendo el período de } \\
\text { cambio. }\end{array}$ \\
\hline Paciente encamado & $\begin{array}{l}\text { Deambulación afectada } \\
\text { relacionada a perjuicio } \\
\text { cognitivo, neuromuscular y } \\
\text { musculo-esquelético y } \\
\text { fuerza muscular insuficiente. }\end{array}$ & $\begin{array}{l}\sigma \text { Verificar a integridad de la piel, } \\
\text { utilizando hidrocoloide si } \\
\text { necesario. } \\
\sigma \text { Realizar masaje con AGE para } \\
\text { mejorar la circulación. } \\
\text { Orientar en cuanto a la } \\
\text { importancia del cambio de } \\
\text { decúbito. } \\
\sigma \text { Colocar almohadas para evitar }\end{array}$ \\
\hline
\end{tabular}




\begin{tabular}{|c|c|c|}
\hline & & $\begin{array}{lll}\text { presión } & \text { sobre } & \text { nervios } \\
\text { superficiales. } & & \end{array}$ \\
\hline Dolor crónico & $\begin{array}{l}\text { Dolor crónico } \\
\text { relacionado con incapacidad } \\
\text { física crónica. }\end{array}$ & $\begin{array}{l}\text { Verificar a intensidad del dolor, } \\
\text { utilizando la escala de dolor. } \\
\text { Promover estrategias para } \\
\text { control del dolor (técnicas de } \\
\text { relajamiento; masajes). } \\
\text { Administrar analgésicos, } \\
\text { conforme prescripción médica. } \\
\text { Usar coberturas apropiadas } \\
\text { que minimicen el trauma. }\end{array}$ \\
\hline Ansiedad & $\begin{array}{l}\text { Ansiedad relacionada con } \\
\text { estado de salud afectado }\end{array}$ & $\begin{array}{l}\sigma \text { Explicar los procedimientos a } \\
\text { ser realizados. } \\
\sigma \text { Ofrecer apoyo psicológico al } \\
\text { paciente y a la familia. } \\
o \text { Proporcionar medidas que } \\
\text { disminuyan la ansiedad. }\end{array}$ \\
\hline Auto-imagen alterada & $\begin{array}{l}\text { Disturbio en la imagen } \\
\text { corporal relacionado con } \\
\text { lesión. }\end{array}$ & $\begin{array}{l}\text { Desarrollar una reintegración } \\
\text { de la imagen corporal. } \\
\text { Estimular la verbalización de } \\
\text { sentimientos. } \\
\text { o Ofrecer ayuda al paciente para } \\
\text { promoción de la salud. }\end{array}$ \\
\hline
\end{tabular}

\section{Evolución de enfermería}

\section{or 11/03/2010}

J.V.S., 83 anos, sexo masculino, $14^{\circ}$ día de internación hospitalaria (DIH) para limpieza quirúrgica en MII. Despierto, consciente. Normocárdico, normotenso, afebril, eupneico. Respiración espontánea, sin $\mathrm{O}_{2}$ suplementario. Ausculta pulmonar y cardíaca fisiológica. MMSS resecados, hipohidratados, perfusión tisular periférica mantenida, ausencia de venoclisis. Acepta dieta. Abdomen plano, indoloro a la palpación. Diuresis espontánea reducida. Evacuaciones presentes. Renovado curativo en pie izquierdo: herida extensa, profunda, pequeños puntos de fibrina, amplia área de granulación, exudado seroso, moderada cantidad. Utilizado alginato de calcio + AGE + SF al 0,9\% + tela no adherente. Cambiar en 48 horas y si necesario cambiar curativo secundario.

\section{$16 / 03 / 2010$}

$19^{\circ} \mathrm{DIH}$. Renovado curativo en pie izquierdo de úlcera venosa: herida extensa, profunda, pequeñas áreas de fibrina en la región periférica, amplia área de granulación al centro, presencia de exudado seroso. Utilizado alginato de calcio + tela no adherente + AGE + SF al 0,9\%. Cambiar en 72 horas y si necesario, cambiar curativo secundario. Deberá ser encaminado para evaluación del cirujano plástico para injerto cutáneo. 


\section{CONSIDERACIONES FINALES}

Frente a lo expuesto, se observa que la enfermería tiene un papel fundamental en los cuidados al paciente portador de úlcera venosa, ya que constituye una enfermedad crónica, con recuperación lenta y posibilidad de recidivas, con implicaciones importantes en la vida del paciente.

La educación en salud es de suma importancia para este paciente, pues necesita orientación en cuanto a modificaciones en su estilo de vida, implicando cambios en relación a los hábitos alimentarios, al sedentarismo y al hábito de fumar.

Algunos aspectos deben ser enfocados en el tratamiento de úlceras venosas, especialmente el uso continuo de medias de compresión por los pacientes, que deben ser orientados a seguir de forma completa también el tratamiento medicamentoso.

El enfermero educador posibilita a su educando medidas que proporcionen la liberación del paciente para el autocuidado. Es esencial que la enfermería actúe no solo en el cuidado de la herida, sino de forma holística, viendo al paciente de forma global.

La orientación al paciente por parte del enfermero contribuye de forma muy significativa a la busca de estrategias que ayuden a la prevención de esta enfermedad, sus complicaciones y la promoción de la salud, alcanzando, así los pacientes portadores de úlcera venosa, una mejor calidad de vida.

\section{REFERENCIAS}

1 Jorge AS, Dantas SRPE. Abordagem multiprofissional do Tratamento de Feridas. In: Paranhos WY. Úlceras de Pressão. São Paulo: Atheneu, 2005.

2 Franco D, Gonçalves LF. Feridas cutâneas: a escolha do curativo adequado. Revista Col. Bras. 2008; 35 (3): 203-206.

3 Hess CT. Tratamento de Feridas e Úlceras. Rio de Janeiro: Reichmann \& Affonso, 2002, 4⿳⺈ ed.

4 Carmo SS. et al. Atualidades na assistência de enfermagem a portadores de úlcera venosa. Revista Eletrônica de Enfermagem 2007; 9 (2): 506- 517.

5 Abbade LPF, Lastórias S. Abordagem de pacientes com úlcera da perna de etiologia Venosa. An. Brasileira de Dermatologia 2006; 81 (6): 509-22.

6 Borges EL, Caliri MHL, Haas VJ. Revisão sistemática do tratamento tópico da úlcera venosa. Revista Latino-americana de Enfermagem 2007; 15 (6): 1163-1170.

7 Bersusa AAS, Lages JS. Integridade da pele prejudicada: identificando e diferenciando uma úlcera arterial e uma venosa. Revista Ciência, Cuidado e Saúde 2004; 3 (1): 81-92.

8 Sousa CAC, Santos I, Silva LD. Apropriação das concepções de Neuman e Braden na prevenção de úlceras de pressão. Revista de Enfermagem da UERJ 2004; 1(12): 280-5.

9 Rodrigo J. Estudo de caso: fundamentação Teórica. Brasília: 2008.

10 Alfaro-Lefevre R. Aplicação do processo de enfermagem: um guia passo a passo. Porto Alegre: Artes Médicas, 2005.

11 NANDA. Diagnósticos de enfermagem da NANDA: definições e classificação 20072008. Porto Alegre: Artmed, 2008. 
12 BRASIL. Conselho Nacional de Saúde. Resolução no 196/96. Decreto no 9.333 de janeiro de 1987. Estabelece critérios para pesquisa envolvendo seres humanos. Bioética 1996, 4(2): 15-25. 